\author{
EVS27 \\ Barcelona, Spain, November 17-20, 2013
}

\title{
Smart and Green ACC series: A city and highway specific approach towards a safe and efficient eDAS
}

\author{
Sagar Akhegaonkar ${ }^{1}$, Sebastien Glaser $^{2}$, Lydie Nouveliere ${ }^{2}$, Frederic Holzmann ${ }^{1}$ \\ ${ }^{1}$ INTEDIS GmbH \& Co. KG, Max-Mengeringhausen-Straße 5, 97084 Wuerzburg - Germany (phone: +49 (0) 931 \\ 6602-35515; e-mail: sagar.akhegaonkar@intedis.com).
}

${ }^{2}$ LIVIC (Laboratory on Interactions Vehicles-Infrastructure-Drivers), a research unit of IFSTTAR, Batiment 824, 14, Route de la Miniere, 78000 Versailles, France (phone: +331404329 08; e-mail: sebastien.glaser@ifsttar.fr).

\begin{abstract}
The successful transition of fully electric vehicle into automotive market is plagued with expensive product prices and limited drive range. While manufacturers point to fuel saving benefits, the actual cost savings after the first battery replacement presents negative economics. Hence it is necessary to maximise the fuel saving costs and to prolong the battery life as much as possible. The situation calls for an assistant system which takes into consideration the inherent propulsion system dynamics of electric vehicle in two typical situations - namely city and highway. Here we propose a combination of two systems, first a dynamic programming based acceleration controller for city cycle and second, an eHorizon based ACC system for maximum recuperation on highways. This paper is an extension of papers [1,2] and forms a series which is attributed to the development of a partial or complete "Safe and energy efficient longitudinal vehicle controller". Such a controller is named "SAGA" - Smart and Green Automated Cruise Control. It is an ecological driver assistance system (eDAS) that adapts the vehicle speed over all its speed range according to a forward vehicle and to road events in a near horizon (legal speed, curves, etc...) with an aim to reduce the energy consumption without compromising on safety.
\end{abstract}

Keywords: Battery electric vehicles, Driver Assistance systems, Energy management.

\section{Introduction}

The battery electric vehicle has been here for over a century. It once shared the commercial market with its internal combustion and steam engine contemporaries. However, due to its limited range and the advent of cheap oil, electric vehicle technology was overlooked for better and more convenient internal combustion engine vehicles. Recently, with concerns regarding environmental pollution and limited oil reserves, automotive industry is again looking towards electric vehicles. While battery electric vehicles
(BEV) offer transportation by means of clean energy, they are still at a disadvantage due to their limited driving range. Moreover, even with substantial research and development, the battery remains the single most expensive component which is prone to degradation over time. The overall costs of owning a BEV are closely related to the capital, depreciation and replacement cost of the battery itself. Hence it is of paramount importance to ensure that the powertrain of a BEV runs as efficiently as possible without overstressing its components.

Normally, vehicle powertrains are subjected to two different operating environments, namely, city and 
highway which are characterised with parameters like acceleration, velocity and braking frequencies. The city cycle consists of frequent acceleration and braking events and has a comparatively low average velocity. In contrast the highway cycle is characterised with less frequent, low value acceleration and braking but a high average velocity. The BEV powertrain operates in two completely different domains in the city and highway environment. These domains differ with respect to the overall efficiency of the powertrain and also the maximum recuperation torque capacity. Hence two different approaches are taken in order to develop the ecological driving assistance system (eDAS) for BEVs.

In the second section of the paper, a brief overview of BEV model is presented. This model is used to simulate the results for the energy management strategies. Section three is dedicated to the problem definition, simulation and results for the Dynamic programming based acceleration controller strategy for city traffic conditions. Furthermore, the next section presents the Smart and Green ACC function (SAGA) for the longitudinal controller applied to the highway traffic environment. Finally a brief discussion concludes the paper.

\section{Battery Electric Vehicle model}

A detailed description of the model used for simulation can be found in [1]. The BEV model is developed in Matlab®, Simulink® environment. The TATA eVista prototype, which is used in the eFuture project, is considered as a base for model development. Various modelling parameters relevant to the eVista are listed in Table 1.

Table 1: Vehicle Parameters

\begin{tabular}{lcc}
\hline Vehicle mass & $m$ & $1550 \mathrm{~kg}$ \\
Front area & $A$ & $2.28 \mathrm{~m}^{2}$ \\
Drag co-efficient & $C_{w}$ & 0.36 \\
Wheel radius & $r$ & $0.3 \mathrm{~m}$ \\
$\begin{array}{l}\text { Co-efficient of rolling } \\
\text { resistance }\end{array}$ & $f_{r}$ & 0.015 \\
\end{tabular}

The BEV model is developed with an aim to calculate the energy consumption and mainly takes into account the forward longitudinal movement of the vehicle. The following ideology is used to calculate the energy.

$$
\begin{aligned}
E_{x} & =\int P_{x} d t \\
P_{x} & =\int F_{x} d v
\end{aligned}
$$

$F_{x}=\left(\frac{C_{w} \cdot \rho \cdot A \cdot v^{2}}{2}+M_{v} \cdot g \cdot f_{r}+M_{v} \cdot g \cdot \sin \beta+M_{v} \cdot a\right)$

Table 2 lists the terminology that has been used to define the formulae.

Table 2: Terminology

\begin{tabular}{lcc}
\hline Vehicle mass & $M_{v}$ & $k g$ \\
Front area & $A$ & $m^{2}$ \\
Drag co-efficient & $C_{w}$ & - \\
Wheel radius & $r$ & $m$ \\
Co-efficient of rolling & $f_{r}$ & - \\
resistance & & $\mathrm{m} / \mathrm{s}^{2}$ \\
Vehicle acceleration & $a$ & $\mathrm{~m} / \mathrm{s}$ \\
Vehicle velocity & $v$ & $\mathrm{~J}$ \\
Energy & $E_{x}$ & $\mathrm{~N}$ \\
Force & $F_{x}$ & $\mathrm{~W}$ \\
Power & $P$ & $\mathrm{~kg} / \mathrm{m}^{3}$ \\
Air density & $\rho$ &
\end{tabular}

While the in depth details for the Simulink® model can be found in [1], a brief description of the two core components namely the propulsion motor and the battery is given in the following.

\subsection{The e-Motor}

A $37 \mathrm{~kW}$ PMSM e-Motor with $750 \mathrm{Nm}$ peak torque is modeled. The motor model is based on look up tables which define the speed-torque characteristics and also the power losses corresponding to the operating point of the motor. The look-up tables are generated through physical measurement of the real motor over its operating range (0 - $1070 \mathrm{rpm})$.

The motor model is supplied with speed (w), torque request and battery voltage as inputs. The motor torque is calculated by adding the driver requested torque and power losses. Power required is the product of motor torque and speed. Since the voltage is known the current requirement for this power request is calculated. The calculated current 
value is supplied as an input signal to the battery (ESS) block.

The torque speed characteristics for the e-Motor are shown in Figure1 along with the efficiency map and the maximum possible acceleration (using 2 such motors) for the eFuture project [3] prototype vehicle. Hence a total maximum torque of $1500 \mathrm{Nm}$ can be available. The maximum acceleration is calculated taking into consideration the vehicle data in Table 1 .

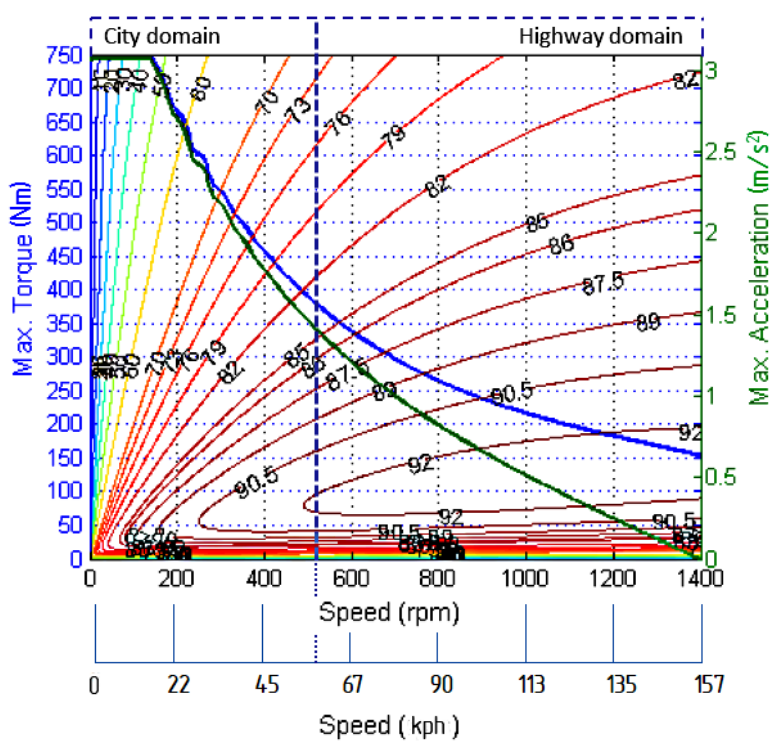

Figure1: Motor torque-speed-efficiency characteristics

As described earlier, the motor map can be divided into two operation domains depending on the speed, namely, city and highway. The motor has an integrated reduction gear. It can be observed that the gearing adjusts the torquespeed characteristics to obtain maximum possible efficiency.

\subsection{The Battery (ESS)}

The Energy Storage System (ESS) is a lithiumion battery developed as a part of the eFuture project by Miljøbil Greenland AS. From the energy calculation point of view, the battery is an energy storage device which supplies or accepts the requested current at the present battery voltage and efficiency. The model is based on a virtual circuit as defined in [4] which represents the battery capacity, internal resistance and transient behaviour of the battery. It is sought to keep a balance between accuracy and model complexity in order to reduce the computational load. Thereby, a temperature dependant internal resistance variation is taken into consideration while calculating the power loss and efficiency.
Figure 2 represents the battery behaviour for a typical city driving cycle.

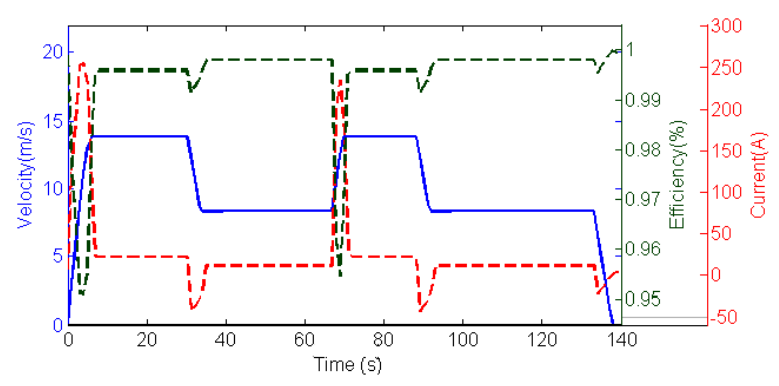

Figure2: Battery characteristics over a velocity cycle

\section{Dynamic programming based acceleration controller for city traffic}

The acceleration controller for the city traffic condition is based on a previous version which is defined in depth in [1]. The new version uses the same basic methodology of dynamic programming [5] but has better controlling variables and hence improved controller behaviour. In the following sections the fundamental idea, problem definition, optimisation procedure and results are described.

\subsection{Effect of vehicle acceleration on total energy consumption}

As already mentioned, the city traffic situation is characterised with frequent starts and stops. A global standard for a speed limit in cities is $50 \mathrm{kph}$, which means that the BEV operating in city domain will perform many acceleration and braking manoeuvres at speeds less than the specified limit. At low speeds, where the wind resistance is small, the major part of energy must be spent to accelerate the vehicle mass. The energy equation for acceleration energy of a vehicle with constant acceleration for a small sample time " $\mathrm{t}$ " can be derived from equations 1,2 and 3 as follows.

$$
\begin{gathered}
F=m \times a \\
P_{\text {acceleration }}=\int F_{a} d v \\
E_{\text {acceleration }}=\int m \times a^{2} \times t d t
\end{gathered}
$$

From the formula for acceleration energy, it is clear that as the acceleration value increases the 
energy required will increase. Furthermore the equation is modified to take distance into account and total energy for different acceleration values but same covered distance is calculated as shown in Figure3. Here the vehicle model is simulated to cover a $100 \mathrm{~m}$ distance from standstill with a constant acceleration.

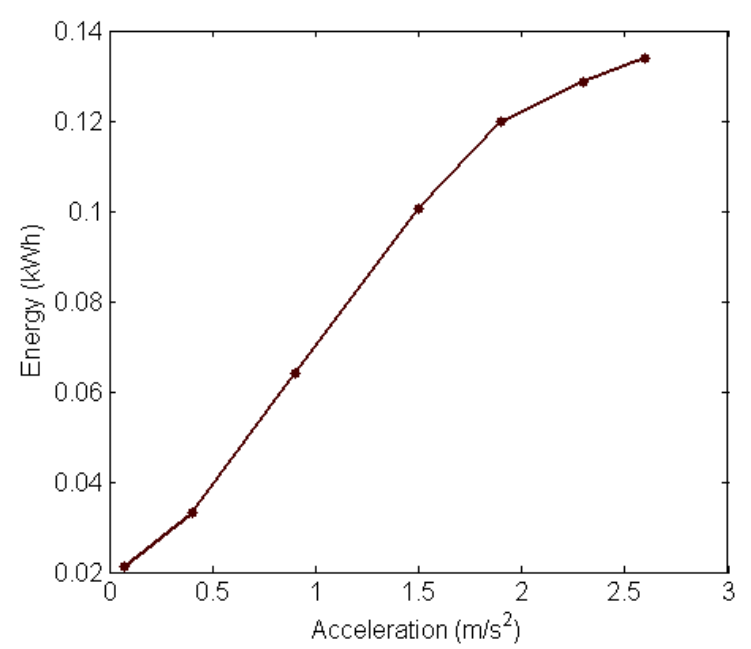

Figure3: Effect of acceleration on Energy consumption

\subsection{Problem definition, optimisation and algorithm development}

\subsubsection{Acceleration phase}

As established in the previous section, reduction in the acceleration value shall reduce the energy consumption. However reduction in acceleration also means more time to cover the same distance. Therefore, an optimum acceleration value must be calculated where, substantial saving of energy can be realised without compromising on the required time for travel.

An algorithm to optimize the energy consumption and time requirement is developed using the classical dynamic programming techniques [1]. This previously developed algorithm takes into consideration the acceleration values as control parameter. However, at higher velocities the motor is not able to supply enough torque to calculate the cost function over the full range of control parameters $\left(0.5 \mathrm{~m} / \mathrm{s}^{2}-2.5 \mathrm{~m} / \mathrm{s}^{2}\right)$. To solve this limitation, the algorithm is modified where now, the maximum torque capacity of motor is taken into consideration as control parameter. Furthermore, the optimization sampling time is modified to take into account the optimum course of driving for the next approximate 2 seconds. This optimal control problem is explained with the help of Figure4. The acceleration phase is discretized in $\mathrm{N}+1$ steps of equal distance from $0-\mathrm{N}$. If $J$ is the objective function which depends on energy and time, then $J$ can be defined as,

$$
J=Q 1 * \text { Energy }+Q 2 * \text { Time }
$$

Where, $Q 1$ and $Q 2$ are the weightage co-efficients. Since $J$ is a function of motor torque " $t$ " of the vehicle where $t \in T$. The control problem can be formulated as follows

$$
\begin{aligned}
& \min \sum_{k=0}^{N-1} J_{k+1}\left(T_{k}\right) \\
& T_{k} \in\left[T_{\text {low }}, T_{\text {high }}\right]
\end{aligned}
$$

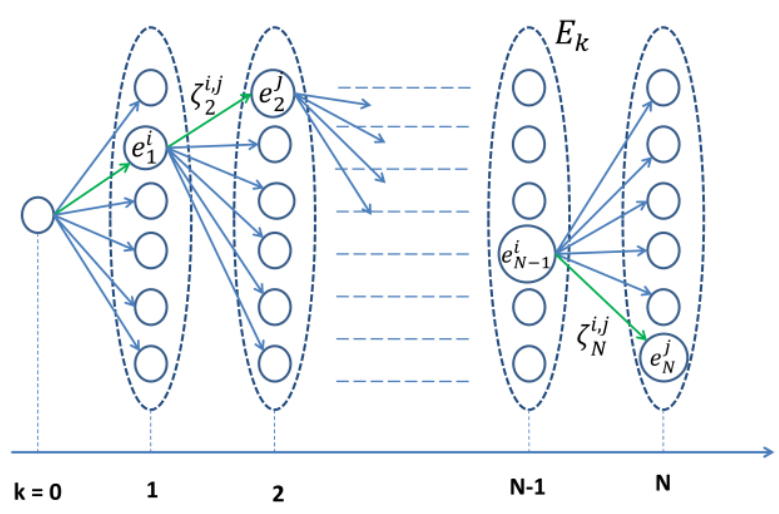

Figure4: Formulation of a DP algorithm

In Figure $4 e_{k}^{i}$ is the state $i$ at the step $k . E_{k}$ is the set of the states $e_{k}^{i}$ at the step $k$ and is defined by the discretized state space $T_{k}$ which is a set of motor torque values from $0.1 * T_{\max } \mathrm{Nm}$ to $T_{\max }$ $\mathrm{Nm}$ with an interval of $0.1 * T_{\max } \mathrm{Nm} . \zeta_{k+1}^{i, j}$ is the cost associated to each transition from the state $i \in E_{k}$ to the state $j \in E_{k+1}$. Thus it can be stated that for a transition from step $k=1$ to $k=2$ where the initial state $e_{1}^{i}$ at step 1 is already defined, the goal is to find a state $e_{2}^{j} \in E_{2}$ at step 2 where the cost of transition $\zeta_{2}^{i, j}$ is minimum. This minimum cost is nothing but $J_{2}\left(T_{1}\right)$ as defined in expression 6. Therefore the dynamic programing technique can be defined as follows. It is partly based on [6].

Let $\pi^{*}=t_{0}^{*}, t_{1}^{*}, \ldots \ldots, t_{N-1}^{*}$ be an optimal control strategy for this problem where " $t$ " is the motor torque value. Suppose that, when torque $t_{k-1}^{*}$ is 
applied, the state $e_{k}^{i}$ is reached at time $i$ during the acceleration phase. Considering the subproblem for which, from the state $e_{k}^{i}$ at time $i$, we seek to minimize the cost to go from $k$ to $N: J=$ $\Sigma_{k}^{N-1} \zeta_{k+1}\left(T_{k}\right)$, then the optimal control strategy $\pi^{*}=t_{0}^{*}, t_{1}^{*}, \ldots \ldots, t_{N-1}^{*}$ is optimal for that subproblem.

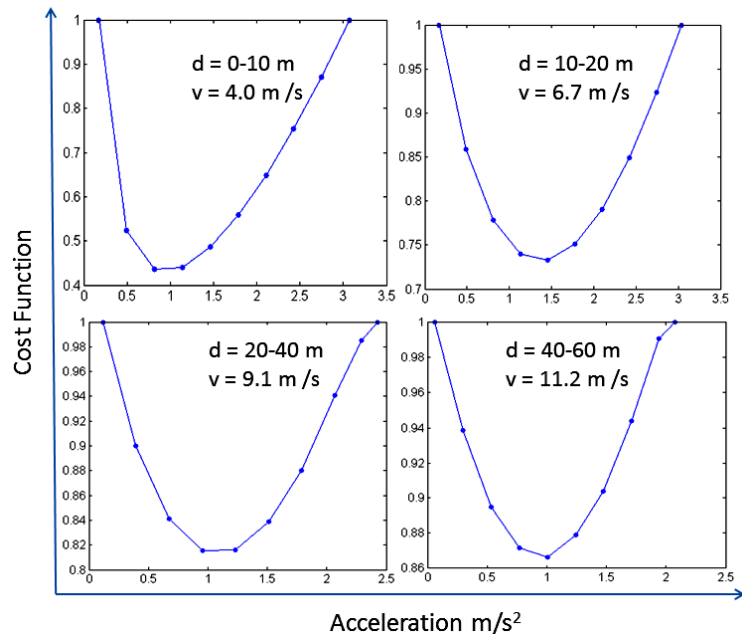

Figure5: Cost function results $(\mathrm{Q} 1=1, \mathrm{Q} 2=1)$

For the transition from every optimum state to the next probable state with a constant applied torque " $t$ ", the initial and final velocities are recorded along with the time. Thus the acceleration for the state transition is calculated. Results for optimum values of acceleration depending on cost function $J$ of energy and time for equal distance steps are shown in Figure5. The cost function is normalized to return a value in range of 0 - 1. Each box represents an optimization process. It can be observed that for a specific sample distance, an optimum acceleration value is obtained. The velocity " $\mathrm{v}$ " in the boxes is the velocity attained by the vehicle when it has covered the given sample distance with the constant optimum acceleration. As the velocity increases the sample distance is also increased so as to optimize approximately 2 seconds of the drive ahead. It can be observed that as the velocity increases the maximum acceleration which corresponds to the control points decreases. Hence a valid spread of the control parameters for calculating the cost function is achieved which was not the case for the previous algorithm [1].

\subsubsection{Deceleration phase}

An energy management strategy, which aims at energy consumption reduction, operates on the basic principle of minimization of energy investment and maximization of energy recuperation. In a BEV, energy can be recuperated by using the propulsion motor as a generator during braking manoeuvres. While propulsion motors can supply strong braking torques, most OEMs use only a part of full motor braking capacity. The previous version of the deceleration phase algorithm [1], which is based on the specifications of the eVista [3] prototype uses only $-260 \mathrm{Nm}$ of braking torque from motor while the remaining is supplied by the service brakes. Any torque supplied from service brakes does not result in energy recuperation and hence this energy is lost. In this paper, a modified version of the algorithm is investigated where, full motor barking capacity is utilised for braking and thereby, a case for exclusive motor braking is presented. Again however, as observed in Figure1 motor torque capacity is a function of motor speed and reduces at higher speeds. Hence to ensure a safe exclusive motor braking, the distance from where braking should be started must be calculated in advance. This concept is explained in Figure6.

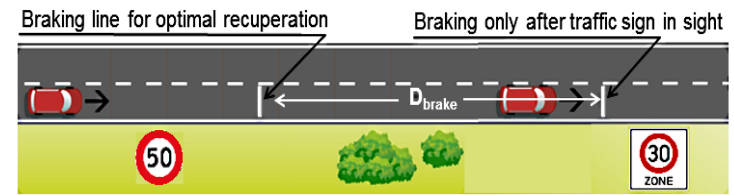

Figure6: Braking manoeuver (Speed limit reduction)

For a vehicle travelling at $50 \mathrm{kph}$ and approaching a speed limit of $30 \mathrm{kph}$, a normal driver will brake only after he observes the traffic sign. However, here he must use the service brakes which provide a strong braking force.

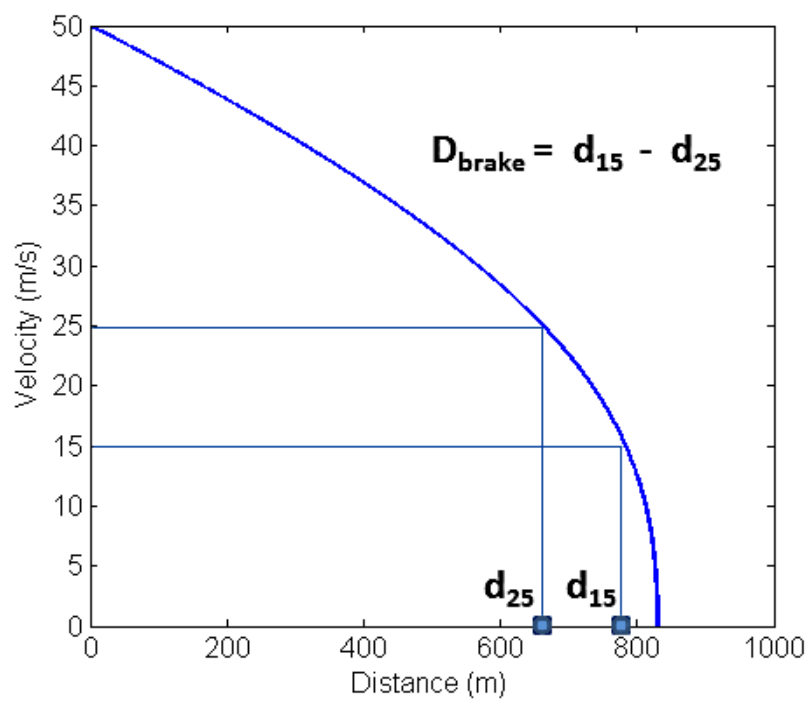

Figure7: Optimum braking distance 
In order to recover all the energy from this braking manoeuver, the motor brake must be used. Since motor brakes are not as strong as service brakes, the braking must commence at a distance $D_{\text {brake. }}$. To calculate this distance at given velocity, the vehicle was simulated to brake from a velocity of $50 \mathrm{~m} / \mathrm{s}$ to $0 \mathrm{~m} / \mathrm{s}$ exclusively under the influence of motor brakes. The distance $\mathrm{D}_{\text {brake }}$ can be calculated from this data as shown in Figure7. Hence, for a vehicle which must brake from $25 \mathrm{~m} / \mathrm{s}$ to $15 \mathrm{~m} / \mathrm{s}$ must start braking at a distance of $d_{15}-d_{25}$. For this purpose, the location where such barking manoeuvers are applicable e.g. speed limit, traffic signal, traffic jams etc. must be known in advance. Such information could be available through digital maps, vehicle to vehicle $(\mathrm{V} 2 \mathrm{~V})$ and vehicle to infrastructure (V2I) communication in future.

\subsection{Simulation and Results}

The final city traffic algorithm combines the strategies for acceleration and deceleration phases as described earlier. In an actual operating mode the vehicle is supposed to follow the optimum acceleration values till it reaches the specified velocity limit, where then the speed is maintained till the optimum braking distance is reached. At this point the motor braking is applied. The system behaviour is presented by means of a use case where the algorithm is applied to a city cycle. This cycle is non-standard and consists of defined speed limits at specific distances. Velocity cycles such as NEDC or Artemis are not used since they are more applicable to emission calculation and not the real electric vehicle behaviour.

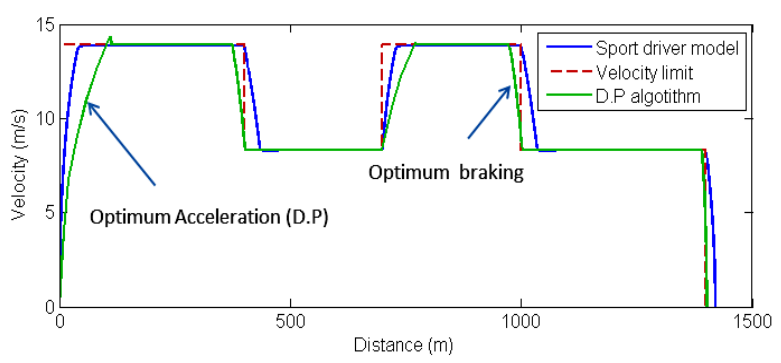

Figure8: Optimum velocity profile (City cycle)

Figure8 compares the dynamic programming based algorithm to a driver model. This driver model is tuned to maximise the performance offered by the vehicle. Therefore its behaviour tends to apply strong acceleration and braking values. It is assumed that the driver only brakes when it notices the traffic signs. In the figure 8 velocity profiles over distance travelled are plotted for two driving styles. It can be observed that the velocity profile for D.P. based algorithm has a gradual increase in velocity compared to a strong acceleration profile of the driver model. The optimum velocity profile balances the energy consumption and time to travel. Also, since the D.P. algorithm strategy already knows about the speed limits beforehand, it can start the optimum exclusive braking manoeuver earlier. Thereby it absorbs all the available braking energy which would normally go waste. As compared to the previous algorithm [1], the use of maximum motor capacity for braking has resulted into comparable barking performance to driver model which is designed to brake with a $-750 \mathrm{Nm}$ torque. The total cycle distance is $1400 \mathrm{~m}$ which is covered by the driver model in $140 \mathrm{sec}$ and the D.P. algorithm in $145 \mathrm{sec}$. As compared to the previous algorithm [1], the modified algorithm requires much less time to cover the same cycle because of the increased braking capacity. It is assumed that the driver model can recuperate with $-260 \mathrm{Nm}$ (out of total $-750 \mathrm{Nm}$ ) while D.P. algorithm uses full motor capacity for braking. While the driver model requires $0.259 \mathrm{kWh}$, the D.P. based controller requires $0.241 \mathrm{kWh}$ for same distance, saving approximately $9 \%$ energy. This figure will change depending on frequencies of acceleration and braking events over the cycle.

\section{Green ACC for highway traffic}

As explained in section 1 and observed in Figure 1 the BEV clearly operates in two separate domains in the city and highway environment. Hence the dynamic programing based acceleration control algorithm applicable to the city traffic cannot be used for speeds exceeding $60 \mathrm{kph}$. As the velocity increases the energy supplied to overcome the air drag increases exponentially. In the highway operation domain, where the power to overcome air drag is greater than the power to accelerate the vehicle, it does not make sense to save on acceleration energy. Here, at higher speeds the main energy recovery potential lies in the momentum of the vehicle. Conventional Adaptive Cruise Control (ACC) systems are designed to use service brakes of a vehicle to provide the necessary reduction in speed. However, in a BEV, where there is possibility to recuperate the braking energy use of service brakes will result in loss of large packets of energy which could have been redirected to the battery. For this purpose it is proposed to apply exclusive motor braking in the highway domain. Again, as pointed out earlier, the 
motor braking is not as strong as service brakes and hence the actual algorithm for the ACC must be modified to take into consideration the motor limitations. The aim of the Green ACC is to recuperate as much as possible energy from the barking manoeuvers to increase the efficiency but without compromising on the safety.

\subsection{ACC operation modes}

Fundamentally there are two situations an ACC must handle. In the first case, there is no vehicle in the front of the ego vehicle which the sensors can sense. Here the ACC must follow the speed set by the driver. This operation mode can be termed as speed control mode. Since no braking manoeuver is involved here, this paper does not focus on the speed control mode. When the ACC sensors detect a slow moving vehicle in front of the ego vehicle, the ACC switches mode to distance control as described in Figure9. When the vehicle in front is detected, a chain of events which is characteristically repetitive follows. First there is a strong braking manoeuver followed by speed equalisation. Once the ego vehicle reaches a specified safe distance behind the front vehicle (safe headway spacing) it must follow the front vehicle while maintain its speed and the headway spacing. The main focus here is on the barking manoeuver where, a large packet of potentially recoverable energy lies.

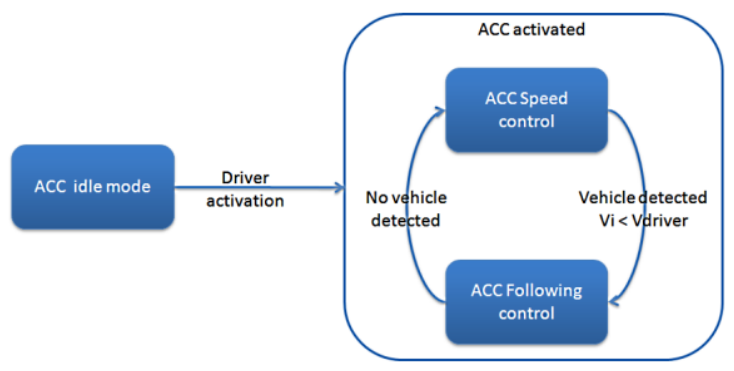

Figure9: ACC operation modes [2]

\subsubsection{Distance control: Problem definition}

Table 3 states the terminology used in context to the ACC system.

Table3: ACC terminology

\begin{tabular}{lcc}
\hline Clearance to the lead vehicle & $d$ & $\mathrm{~m}$ \\
Speed of the ego vehicle & $V$ & $\mathrm{~m} / \mathrm{s}$ \\
Driver desired speed & $V_{d}$ & $\mathrm{~m} / \mathrm{s}$ \\
Time headway & $T$ & $\mathrm{~s}$ \\
Desired time headway & $T_{d}$ & $\mathrm{~s}$ \\
\hline
\end{tabular}

\begin{tabular}{lcc}
\hline Speed of lead vehicle & $V_{i}$ & $\mathrm{~m} / \mathrm{s}$ \\
Relative speed & $\Delta V$ & $\mathrm{~m} / \mathrm{s}$ \\
\hline
\end{tabular}

In the distance control mode the objective is to regulate the error on the headway clearance $e_{d}$ $\left(e_{d}=d-T_{d} V\right)$ around 0 . The algorithm can be made more robust by including the relative speed $\Delta V$ into control equation. The resulting acceleration is control output defined by these two errors. However at higher speeds the motor braking capacity decreases as seen in Figure9 where, the deceleration is calculated taking into consideration the air drag and wheel resistance.

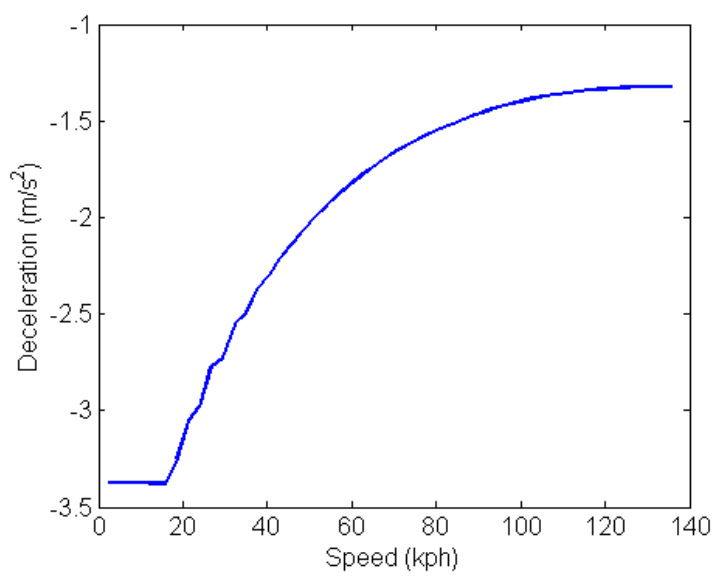

Figure10: Deceleration capacity of motor

Hence it may not be possible to ensure the same safety level as in Conventional ACC strategy. The main reasons for this are, first, during the initial braking manoeuver if the required deceleration is more than the maximum motor braking capacity, then surely, collision cannot be avoided. And second, during the headway distance following mode, if the front vehicle undergoes very strong deceleration then it may not be possible to equalise the speeds only with the help of motor braking. Therefore certain modifications in the original ACC algorithm are suggested in [2]. For an extreme use case where initial time headway is $2 \mathrm{~s}$, ego vehicle speed is $30 \mathrm{~m} / \mathrm{s}$, and front vehicle speed is $7 \mathrm{~m} / \mathrm{s}$, the possible solutions are:

- To increase the minimal time headway that is defined by the driver. However, to obtain the same safety, we need to increase the minimal time headway up to $5 \mathrm{~s}$. The resulting distance is hardly achievable as the sensor range is limited and other road user may cut in the space between vehicles.

- To switch to conventional braking if the time headway drops below a given threshold. For instance, we can maintain a collision free 
system, as for a conventional ACC, with initial time headway of $3.7 \mathrm{~s}$ and an activation of a stronger braking at a threshold on the time headway of $1.5 \mathrm{~s}$

- To switch to an emergency braking if the deceleration of the lead vehicle and the distance drops below given thresholds. If the emergency braking can generate a deceleration of $-6 \mathrm{~m} / \mathrm{s}^{2}$ when the time to collision (difference of distance divided by the difference of speed) is below $2 \mathrm{~s}$.

While [2] investigates further with the second solution, this paper will focus with the third solution. Having a headway spacing of $3.7 \mathrm{~s}$ at a speed of $30 \mathrm{~m} / \mathrm{s}$ means, the gap between two vehicles is $90 \mathrm{~m}$. This is a large gap and there is a high probability of vehicle cut in. Therefore, here we investigate whether a headway spacing of $2 \mathrm{~s}$ can be maintained without compromising safety by use of emergency brakes.

\subsection{Simulation and results}

For simulation of a Green ACC (GACC), to compare it with conventional ACC strategy, a use case is specified. Here we define the lead vehicle velocity is $10 \mathrm{~m} / \mathrm{s}$, ego vehicle is travelling at $20 \mathrm{~m} / \mathrm{s}$ and the desired velocity set point is at $30 \mathrm{~m} / \mathrm{s}$. The headway spacing is set at $2 s$ to avoid vehicle cut in. Sensor sensing distance is $150 \mathrm{~m}$. This can be considered as an extreme case scenario where the resulting velocity difference will be of $20 \mathrm{~m} / \mathrm{s}$ (from 30 to $10 \mathrm{~m} / \mathrm{s}$ ). Also vehicle which drives at $10 \mathrm{~m} / \mathrm{s}$ on highways is an exaggeration to demonstrate that even in extreme cases the safety is not compromised.

It is observed from Figure11 that as far as driver comfort is concerned the Green ACC does not offer degraded performance as compared to the conventional ACC. The deceleration phases starts for both the ACCs at the same time, while Green ACC manages speed equalisation earlier. The only difference is that the major part of deceleration is performed by GACC in the later phase as compared to ACC. It must be stated that behaviour of ACC or GACC would change depending on the gain factors corresponding to the error $\left(\Delta \mathrm{V}\right.$ and $\left.e_{d}\right)$ input to the controller. Here the gain factors are chosen to maximise safety in GACC and same factors are then applied to ACC for a fair comparison. The Green ACC uses the motor braking over the complete range of braking manoeuver and hence manages to recover most of the energy.
From the simulations it is found that beyond a velocity difference of $20 \mathrm{~m} / \mathrm{s}$ it is not safe to use exclusive motor braking and hence it is recommended to initially switch to conventional braking and then to GACC mode after the relative speed is reduced to safe domain of GACC.

Also during the headway spacing following mode, if the spacing falls below $1.5 \mathrm{~s}$, the control is switched to conventional ACC. This use case is explained in [2] where a safe recovery and reswitch to GACC above 2 s spacing is demonstrated.
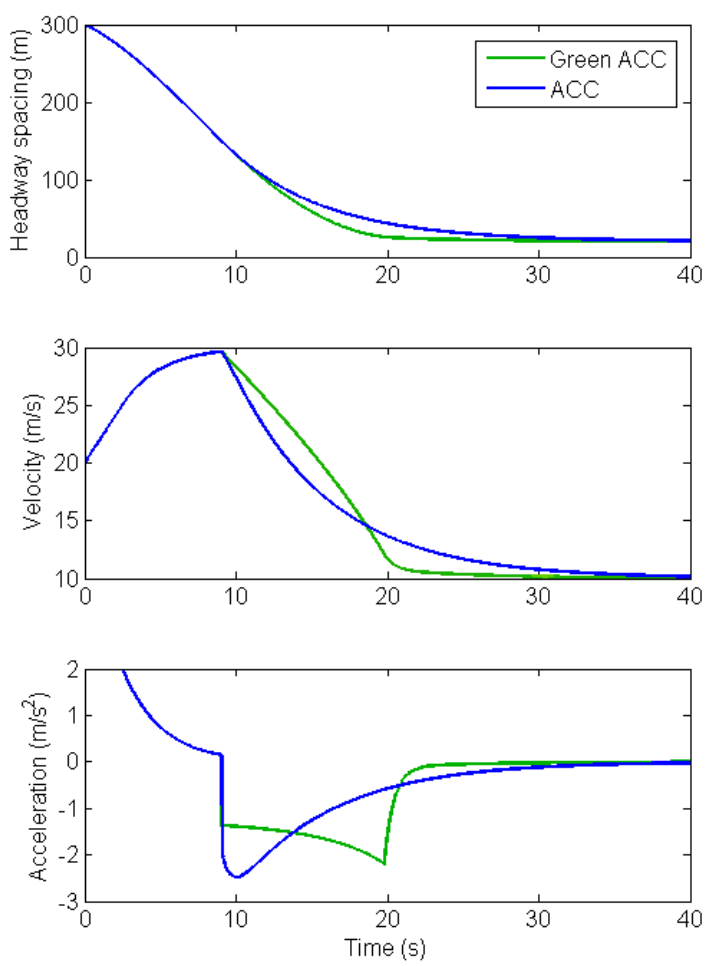

Figure11: GACC highway controller

\section{Conclusion}

Development of a Smart and Green Adaptive cruise controller is a multistep process. In this paper we have identified two operating domains of the battery electric vehicle propulsion system for the city and highway operating conditions. As established, the characteristics of these domains are completely different and hence the same strategy cannot be applied. For city traffic conditions where there is frequent acceleration and braking, the dynamic programming based controller is investigated. From the results it can be seen that the new modified algorithm has a comparable time of travel to an aggressive driver model but also manages to save $9 \%$ energy. However more than energy saving, it is important that since the optimum acceleration values 
provided by the algorithm are low, it puts less stress on the propulsion system. A lower value of power is being drawn from the battery means that the chemical cycle of the battery is put through less stress. Hence the battery life is not reduced as rapidly as compared to a high acceleration driving behaviour. The main problem here is the driver acceptability to the restriction on drivability. Hence for drivers who would rather like to have high acceleration behaviour, levels of restrictions like efficiency mode, normal mode and sport mode could be developed.

Present status for BEVs does not allow much highway driving due to driving range limitations. However development of vehicles like Tesla Model S does project a trend for BEVs which will be drivable on highways as well. For highway domain, where velocity is high, saving of acceleration energy does not make sense. A different kind of ACC controller is investigated which optimises safety and efficiency in the highway driving conditions. Such a controller is named as Green ACC. The next step would be to integrate these two controllers to form a full speed range ACC (FSRA).

\section{Acknowledgments}

The authors would like to acknowledge the support of European Commission, eFuture project.

\section{References}

[1] Sagar Akhegaonkar, Lydie Nouvelière, Sebastien Glaser, "Modeling and Simulation of Battery Electric Vehicle to Develop an Energy Optimization Algorithm", AVEC'12, The 11th International Symposium on Advanced Vehicle Control September 9-12, 2012, Seoul, KOREA.

[2] Sebastien Glaser, Sagar Akhegaonkar, Olivier Orfila, Lydie Nouveliere "Smart And Green ACC, Safety and efficiency for a longitudinal driving assistance" 17th International Forum on Advanced Microsystems for Automotive Applications (AMAA 2013) "Smart Systems for Safe and Green Vehicles" in Berlin, 17-18 June 2013.

[3] http://www.efuture-eu.org/

[4] Knauff M., McLaughlin J., Dafis C., Niebur D., Singh P., Kwatny H., \& Nwankpa C. (2007, May), "Simulink model of a lithium-ion battery for the hybrid power system testbed", In
Proceedings of the ASNE Itelligent Ships Symposium.

[5] R. Bellman, "Dynamic Programming", Princeton University Press, Princeton, NJ, 1957.

[6] Hong Tu LUU, Lydie NOUVELIERE and Saïd MAMMAR "Ecological and Safe Driving Assistance System: Design and Strategy", Intelligent Vehicles Symposium (IV), 2010 IEEE pp. 129 - 134.

\section{Authors}

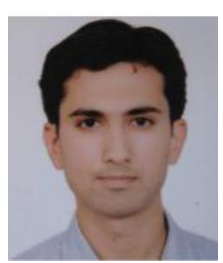

Sagar Akhegaonkar received his Bachelor in Mechanical Engineering from Pune University, India in 2006. He completed M.Sc. in Automotive Engineering from RWTH Aachen University, Aachen, Germany in 2011. $\mathrm{He}$ is presently working in Intedis $\mathrm{GmbH} \& \mathrm{Co}$. KG and affiliated to University of Evry, Paris, France as a $\mathrm{PhD}$ candidate. His research interests include energy optimisation in pure and hybrid electric vehicles.

Sébastien Glaser received the Dipl.Ing. degree from the "Ecole Nationale des Travaux Publics de l'Etat", Lyon, France in 2001 the M.Sc. degree in Computer Vision from the Universite de Saint Etienne the same year and the $\mathrm{Ph} . \mathrm{D}$. degree in automatic control from Université d'Evry, France in 2004. He has been with LCPC Since 2001. He now works as a Senior Researcher at the Département IM, LIVIC French Institute of Science and Technology for Transport, Development and Networks.

Lydie Nouvelière received the M.Sc. degree in Autonomous Robotics from the University of Pierre et Marie Curie in 1999 and the Ph.D. degree in automatic control from the University

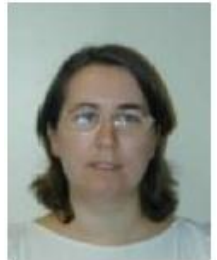
of Versailles, France in 2002. She has been with University of Evry-Vald'Essonne - IBISC Lab., France, since 2003 as associate professor in the department of electrical engineering and scientific advisor at IFSTTAR LIVIC, France, since 2007. 